\title{
Data Privacy, Regulations and Legal Issues on COVID-19 Tracking Apps: A Scoping Review
}

\author{
Utoomporn WONGSIN ${ }^{\mathrm{a}, 1}$, Arnat WANNASRI ${ }^{\mathrm{b}, \mathrm{c}}$, Usman IQBAL $^{\mathrm{d}}$ \\ and Tuo-Yu CHEN ${ }^{\mathrm{d}}$ \\ a PhD student in Global Health and Health Security, Taipei Medical University, Taiwan \\ ${ }^{\mathrm{b}}$ Nopparat Rajathanee Hospital, Department of Medical Services, Ministry of Public Health, \\ Thailand \\ ${ }^{\mathrm{c}}$ National Yang Ming Chiao Tung University, Taiwan \\ ${ }^{\mathrm{d}}$ Masters and PhD Program in Global Health Department, College of Public Health, Taipei \\ Medical University, Taiwan
}

\begin{abstract}
It cannot be deniable that smartphone apps have grown exponentially and are playing a crucial role in the response to the COVID-19 pandemic in many countries. This paper aims to investigate data privacy, regulations and legal issues on COVID-19 tracking apps. A literature search will be followed the PRISMA guidelines extension for a scoping review. The search will be conducted on PubMed and Google Scholar. A total of 38 articles from 7,626 articles were reviewed. Mostly articles report on data privacy. Not many articles report on regulations and legal issues. However, there are many challenges on COVID-19 applications such as security risks, privacy issues, political, ethical, and legal risks, and standardization issues.
\end{abstract}

Keywords. Covid19 Apps, data privacy, legal issues COVID-19, Mobile Application, Global Health, Medical Informatics.

\section{Introduction}

As of 31 January, $2019 \mathrm{nCoV}$ declared global health emergency by World Health Organization (WHO), and WHO named the disease Coronavirus Disease 2019 (COVID19) on 11 February 2020[1-2]. The COVID-19 outbreak has spread worldwide [3], and all attacked countries have been making efforts to control this threat [4]. Since the COVID-19 attacked our world, no-contact tracing using mobile applications has grown exponentially and played a crucial role in the response to the COVID-19 pandemic in several countries.

Mobile apps for COVID-19 are to track and monitor people who are suspected to have COVID-19 infection and then report directly to government agencies. However, there is no evidence to provide a big picture of data privacy, regulation, and legal issues on Mobile application for COVID-19. Therefore, this paper aimed to investigate these aforementioned issues.

${ }^{1}$ Corresponding Author, Utoomporn Wongsin, PhD student in Global Health and Health Security, College of Public Health, Taipei Medical University, Taiwan; Email: d537108007@tmu.edu.tw 


\section{Methods}

\subsection{Search strategy}

The literature search was conducted on PubMed and Google Scholar from February 11, 2020 to December 31, 2020. A literature review was conducted following the PRISMA (Preferred Reporting Items for Systematic Reviews and Meta-Analyses) guidelines extension for a scoping review [5].

\subsection{Eligibility criteria}

The inclusion criteria were: 1) the paper should describe the mobile app clearly, 2) had a clear demonstration concerning data privacy, regulation and legal issues on COVID19 applications, and 3) language is restricted to English language.

\subsection{Study selection}

The relevant keywords were used for a search as following: "COVID-19" "coronavirus", "nCOV19", "contact tracing", "COVID-19 apps", "symptom tracking”, "mobile health application", "mobile apps," "mobile applications," "smartphone apps", "mobile phone apps", "Applications", "App data privacy", "Security", "Regulations", "legal issues on apps", "data privacy apps", "legal issues on COVID-19 tracking apps" "data privacy on COVID-19 tracking apps"

\section{Results}
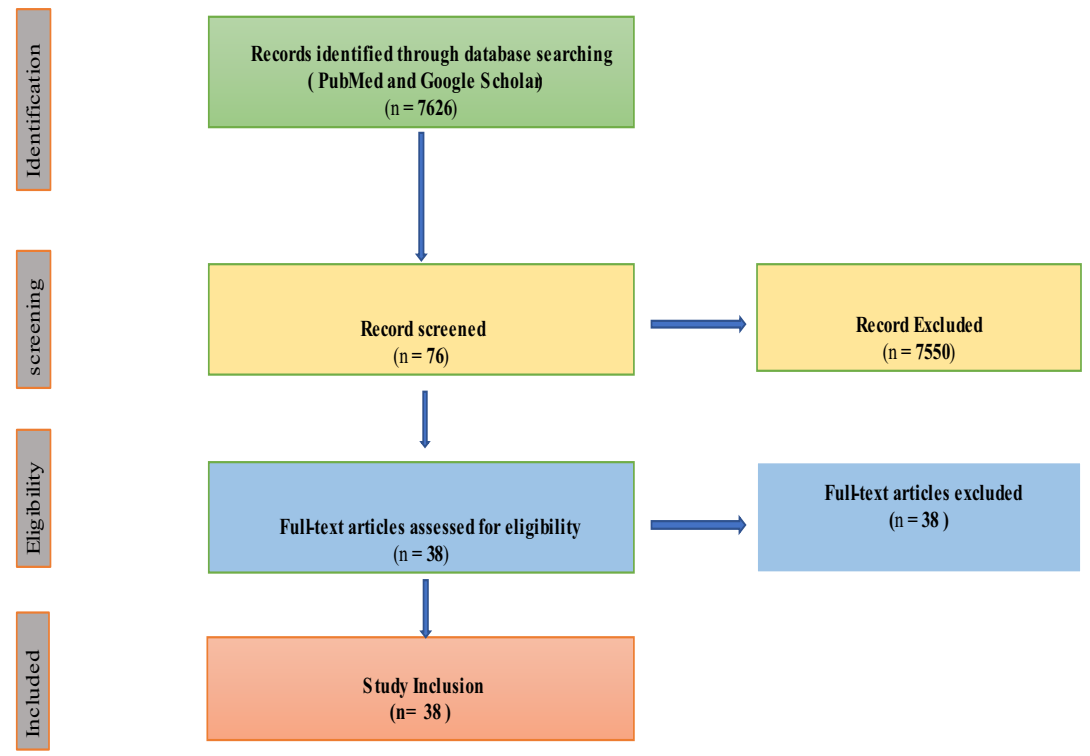

Figure 1. Research Framework according to PRISMA guidelines. 


\subsection{Literature search results}

According to the PRISMA guidelines authors screened the abstracts of all discovered papers and assessed the available full text papers based on the eligibility criteria to finalize the selected papers to be included in the review. A total of 38 articles from 7,626 searched articles were included in this scoping review study.

\subsection{Data privacy}

Currently, it is undeniable that numerous digital technologies for contract tracing are adopted by the government around the globe to combat COVID-19. However, there are several concerns and challenges that needed to be addressed and data privacy is considered as the top issue. Results indicate that data of individuals' privacy were mainly reported (74\% of 38 articles) for disease control policy purpose. Data privacy is considered a backbone of COVID-19 tracking application. However, the data privacy in deploying the applications for COVID-19 tracking are varied from countries to countries. Furthermore, although the protection of individual information privacy and identity is vague, yet the privacy of users should be protected to minimize the privacy concern. This is in line with the standard approach to protect patient's privacy that is to limit access to patients' information [6].

\subsection{Regulation and Legal Issues}

Although the novel contact-tracing technologies have been receiving attention in many countries because of its significant power for combating COVID-19, the applications are supposed to subject to laws and regulations to prevent abuse or exploitation. Findings show that besides data privacy issue, regulations and legal issues were also reported (26\% of 38 articles). Law enforcement should be implemented for data privacy. Although data are necessary for epidemic control, its use should be regulated under data privacy regulations [7]. Individuals' data should be protected by law enforcement agencies.

\section{Discussion}

As the COVID-19 pandemic has drastically worsened, contract-tracing strategy has established as one of the COVID-19-related responses. Mobile applications are the novel powerful tools that were applied in many countries around the world to fight against COVID-19. The government can collect data from the mobile application users to identify those who are potentially exposed to the coronavirus. The contract-tracing using mobile apps have also caused public concerns and doubts on data its intrusion on data privacy. The government should make use of the collected data transparently.

\section{Conclusions}

It can be concluded that, on the one hand, governments use data for controlling the COVID-19 situation, but on the other hand the privacy pertaining to user information 
should be protected by law. To the best of our knowledge, this is the first scoping review on data privacy, regulations and legal issues of COVID-19 tracking apps. Although this study used only two main databases, yet the databases covered an overwhelming number of published papers related to our review issues.

\section{References}

[1] WHO | Pneumonia of unknown cause - China [Internet]. WHO. World Health Organization; [cited 2020 Sep 22]. Available from: http://www.who.int/csr/don/05-january-2020-pneumonia-of-unkowncause-china/en/

[2] Sun P, Lu X, Xu C, Sun W, Pan B. Understanding of COVID-19 based on current evidence. J Med Virol. 2020;92(6):548-551. doi:10.1002/jmv.25722

[3] WHO. Coronavirus disease 2019 (COVID-19): situation report - 78. World Health Organization. 2020. [cited 2020 August 21]. Available from: https:/www.who.int/docs/defaultsource/coronaviruse/situation-reports/20200407-sitrep-78-covid-19.pdf

[4] Mahmood S, Hasan K, Colder Carras M, Labrique A. Global preparedness against COVID-19: we must leverage the power of digital health. JMIR Public Health Surveill. 2020;6(2):e18980. doi: 10.2196/18980. https://publichealth.jmir.org/2020/2/e18980/

[5] Tricco AC, Lillie E, Zarin W, O’Brien KK, Colquhoun H, Levac D, et al. PRISMA Extension for Scoping Reviews (PRISMA-ScR): Checklist and Explanation. Ann Intern Med. 2018 Sep 4;169(7):467-73.

[6] Kasperbauer TJ. Protecting health privacy even when privacy is lost. J Med Ethics. 2020 Nov;46(11):768-72.

[7] Blockchain for COVID-19: Review, Opportunities, and a Trusted Tracking System - PubMed [Internet]. [cited 2021 Jan 10]. Available from: https://pubmed.ncbi.nlm.nih.gov/33072472/ 